\title{
A Phase 2 Study of Concurrent Radiation Therapy, Temozolomide, and the Histone Deacetylase Inhibitor Valproic Acid For Patients With Glioblastoma
}

\author{
Andra V. Krauze ${ }^{\star}$, Sten D. Myrehaug ${ }^{\dagger}$, Michael G. Chang ${ }^{\ddagger}$, Diane J. Holdford ${ }^{\ddagger}$, Sharon \\ Smith ${ }^{*}$, Joanna Shih ${ }^{\star}$, Philip J. Tofilon ${ }^{*}$, Howard A. Fine ${ }^{\S}$, and Kevin Camphausen ${ }^{\star}$ \\ *Radiation Oncology Branch, National Cancer Institute/National Institutes of Health, Bethesda, \\ Maryland tDepartment of Radiation Oncology, Lakeridge Health Durham Regional Cancer \\ Centre, Oshawa, Ontario, Canada $¥$ Massey Cancer Center Virginia Commonwealth University, \\ Richmond, Virginia §New York University Langone Medical Center, New York, New York
}

\section{Abstract}

Purpose-Valproic acid (VPA) is an antiepileptic agent with histone deacetylase inhibitor (HDACi) activity shown to sensitize glioblastoma (GBM) cells to radiation in pre-clinical models. We evaluated the addition of VPA to standard radiation therapy (RT) and temozolomide (TMZ) in patients with newly diagnosed GBM.

\begin{abstract}
Methods and Materials-Thirty-seven patients with newly diagnosed GBM were enrolled between July 2006 and April 2013. Patients received VPA, $25 \mathrm{mg} / \mathrm{kg}$ orally, divided into 2 daily doses concurrent with RT and TMZ. The first dose of VPA was given 1 week before the first day of RT at 10 to $15 \mathrm{mg} / \mathrm{kg} / \mathrm{day}$ and subsequently increased up to $25 \mathrm{mg} / \mathrm{kg} /$ day over the week prior to radiation. VPA- and TMZ-related acute toxicities were evaluated using Common Toxicity Criteria version 3.0 (National Cancer Institute Cancer Therapy Evaluation Program) and Cancer Radiation Morbidity Scoring Scheme for toxicity and adverse event reporting (Radiation Therapy Oncology Group/European Organization for Research and Treatment).
\end{abstract}

Results-A total of $81 \%$ of patients took VPA according to protocol. Median overall survival (OS) was 29.6 months (range: 21-63.8 months), and median progression-free survival (PFS) was 10.5 months (range: 6.8-51.2 months). OS at 6, 12, and 24 months was $97 \%, 86 \%$, and $56 \%$, respectively. PFS at 6,12 , and 24 months was $70 \%, 43 \%$, and $38 \%$ respectively. The most common grade 3/4 toxicities of VPA in conjunction with RT/TMZ therapy were blood and bone marrow toxicity (32\%), neurological toxicity (11\%), and metabolic and laboratory toxicity (8\%). Younger age and class $\mathrm{V}$ recursive partitioning analysis results were significant for both OS and PFS. VPA levels were not correlated with grade 3/4 toxicity levels.

Reprint requests to: Kevin Camphausen, Radiation Oncology Branch, National Cancer Institute, 10 Center Dr, Bldg 10, CRC, Rm B2-3561, Bethesda, MD 20892. Tel: (301) 496-5457; camphauk@ mail.nih.gov.

Conflict of interest: none.

Supplementary material for this article can be found at www.redjournal.org. 
Conclusions-Addition of VPA to concurrent RT/TMZ in patients with newly diagnosed GBM was well tolerated. Additionally, VPA may result in improved outcomes compared to historical data and merits further study.

\section{Introduction}

Primary brain tumors represent $2 \%$ of tumor subtypes; 23,000 new cases and 14,000 deaths per year were registered in the United States, with grade 4 glioblastoma (GBM) being the most common. Standard therapy consists of maximal safe resection followed by concurrent radiation therapy (RT) and temozolomide (TMZ), followed by adjuvant TMZ, which results in an overall survival (OS) of $27.2 \%$ at 2 years and $9.8 \%$ at 5 years (1). Although the efficacy of this therapy remains limited, attempts to increase the effectiveness of the RT/TMZ protocol $(2,3)$ have not been successful.

The pattern of recurrence following combined RT/TMZ therapy indicates failure in or adjacent to the initial RT treatment volume, suggesting that enhancing the effectiveness of RT could lead to an improved therapeutic response. A number of strategies for modifying the delivery of RT (4-6) have been tested, without an improvement in survival. Integration of cytotoxic agents as radiation modifiers into GBM treatment protocols (7) has been disappointing. An increased understanding of the mechanisms mediating radiation-induced cell death has led to the use of molecularly targeted agents such as histone deacetylase inhibitors (HDACi) $(8,9)$. HDACs comprise a family of enzymes that remove acetyl groups from histones as well as other nuclear and cytoplasmic proteins. Inhibition of HDAC activity has been shown to selectively increase tumor cell radiation sensitivity in a variety of in vitro models and to enhance radiation-induced growth delay of subcutaneous human tumor xenografts (8-10). HDACi also reduce the repair of DNA double-strand breaks, a process critical to radiation-induced cell death and consistent with radiation sensitization ( 8 , 9).

Valproic acid (VPA), a nonhepatic enzyme-inducing antiepileptic drug is of particular relevance because it enhances the radiation sensitivity of tumor cells by using in vitro and in vivo model systems $(9,11)$; it is orally bioavailable; it effectively crosses the blood-brain barrier; and its sera and plasma levels are routinely measured as an antiseizure drug (12). On the basis of the preclinical evidence of VPA as a radiation sensitizer and its reportedly safe long-term use as an antiepileptic agent, we initiated a phase 2 study designed to investigate the safety, tolerability, and effectiveness of concomitant RT/TMZ therapy and relatively high-dose VPA followed by adjuvant TMZ in patients with newly diagnosed GBM.

\section{Methods and Materials}

\section{Patient eligibility}

This open-label, phase 2 study XXX was conducted at the XXX and XXX in patients with histologically confirmed GBM, who were 18 years of age or older, and had a life expectancy greater than 8 weeks, and who had undergone surgery no more than 6 weeks prior to enrollment. Pathology review was obtained in all patients. Patients were required to have a Karnofsky performance status $\succ 60$ and adequate hematological, renal, and hepatic function. 
Exclusion criteria included previous VPA therapy, chemotherapy, or RT treatment; a known disorder of urea metabolism; and any history of a second malignancy other than nonmelanoma skin cancer or cervical cancer $<3$ years since diagnosis. Concurrent use of sulfamethoxazole, salicylates, or naproxen was not allowed. The protocol was reviewed and approved by the XXX Institutional Review Board, and written, informed consent was signed by all patients.

\section{Treatment}

Patients received VPA, $25 \mathrm{mg} / \mathrm{kg}$ orally, divided into 2 daily doses, concurrent with RT and TMZ. No VPA was administered adjuvantly. A dose of $25 \mathrm{mg} / \mathrm{kg}$ was selected based on preclinical pharmacology (9). In mice, $150 \mathrm{mg} / \mathrm{kg}$ VPA would yield plasma levels of $\approx 225$ $\mu \mathrm{g} / \mathrm{mL}$ and brain levels of $\approx 60 \mu \mathrm{g} / \mathrm{g}$ of brain tissue (12); and in humans, $25 \mathrm{mg} / \mathrm{kg}$ would yield a plasma level of $\approx 125 \mu \mathrm{g} / \mathrm{mL}$ and brain levels of $\approx 40 \mu \mathrm{g} / \mathrm{g}$. Because $25 \mathrm{mg} / \mathrm{kg}$ of VPA can be given safely in patients with acute mania, this dose was chosen for this study. The first dose of VPA was given 1 week before the first day of RT at 10 to $15 \mathrm{mg} / \mathrm{kg} / \mathrm{day}$ and subsequently increased up to $25 \mathrm{mg} / \mathrm{kg} / \mathrm{day}$, as recommended to reduce side effects, over the week prior to RT (Fig. 1). RT was delivered using 3-dimensional (3D) conformal RT or intensity modulated RT technique 5 days per week in 2-Gy fractions to $60 \mathrm{~Gy}$ total. T2-weighted magnetic resonance imaging (MRI)-defined tumor volume and surrounding edema with a 2-cm margin received $46 \mathrm{~Gy}$. T1-weighted MRI-defined tumor volume with a $2.5-\mathrm{cm}$ margin received an additional $14 \mathrm{~Gy}$. TMZ was given daily at a dose of $75 \mathrm{mg} / \mathrm{m}^{2}$ concurrently with RT. Patients used inhaled pentamidine for PCP prophylaxis. Adjuvant TMZ was given for 5 days at $150 \mathrm{mg} / \mathrm{m}^{2}$ for 1 cycle and then $200 \mathrm{mg} / \mathrm{m}^{2}$ every 4 weeks if tolerated and no tumor progression was detected.

\section{Patient evaluation}

Patients were evaluated at baseline, then weekly while taking RT/TMZ/VPA therapy, and subsequently at 1 month and then 3-month intervals for the first 2 years and then every 6 months for the next 3 years. Evaluations included history and physical examination, hematological and clinical chemistry examinations, and MR imaging. VPA-and TMZrelated acute toxicities were evaluated using Common Toxicity Criteria version 3.0 (National Cancer Institute Cancer Therapy and Evaluation Program), and the Radiation Morbidity Scoring Scheme was used for adverse event reporting (Radiation Therapy Oncology Group [RTOG]/European Organization for Research and Treatment of Cancer [EORTC]). Expected side effects of RT to the brain were not considered adverse events. Any patient with grade 4 adverse toxicity due primarily to VPA or TMZ was removed from the study, as was any patient with grade 3 or 4 nonhematologic toxicity requiring discontinuation of VPA. Treatment response was analyzed according to Response Evaluation Criteria in Solid Tumors (RECIST) (13) criteria and retrospectively by RANO criteria (14). Time to progression was determined from initiation of treatment on protocol to symptomatic or radiographic progression. OS was determined from initiation of treatment on protocol to date of death. 


\section{Statistical analysis}

This prospective phase 2 study was designed to test whether VPA in conjunction with concurrent RT and TMZ therapy improved OS and PFS. The original study design sample calculation was based on data from a study by Stupp et al (15) with a 1-year OS rate of 55\% and a 6-month PFS rate of 55\%. VPA would be considered worthy of future study if the addition of VPA increased 1-year OS and 6-month PFS by approximately $20 \%$. To balance the statistical significance of the trial with the time to accrue an adequate number of patients, we chose 41 patients and an alpha of.06 and a beta of.12 (see Supplementary materials; available online at www.redjournal.com). Kaplan-Meier method was used to estimate OS and PFS. Effects of patient characteristics and clinical factors on OS and PFS were analyzed using univariate Cox proportional hazards model. Age was dichotomized into $\mathbf{5 0}$ years old and $>50$ years old. RTOG recursive partitioning analysis (RPA) was defined according to the method used by Mirimanoff et al (16).

\section{Results}

\section{Patient characteristics}

Forty-three patients were enrolled in this study. Six patients were subsequently ruled ineligible as they did not receive concurrent VPA with RT/TMZ. Thus, 37 patients were included in the analysis. Patient demographics and baseline characteristics are listed in Table 1. Characteristics of the patients enrolled in this study are similar to those in the EORTC 26981/22981-NCIC CE3 trial (1).

\section{Toxicity}

Thirty-seven patients were treated with concomitant RT/TMZ and VPA. All 37 completed the 1-week dose escalation of VPA (Fig. 1). Both TMZ and VPA were discontinued early in 4 patients due to bone marrow suppression (3 patients) and skin reaction (1 patient). Three other patients discontinued VPA but not TMZ due to mental status changes in conjunction with increased ammonia ( 2 patients) or increased amylase ( 1 patient). Four patients discontinued TMZ but not VPA, all due to bone marrow suppression. One patient took TMZ at $100 \mathrm{mg} / \mathrm{day}$, and 1 patient had increased seizure activity resulting in a VPA dosage increase above that prescribed in the protocol. All but 1 patient completed RT as prescribed. That patient developed clinical deterioration and mental status changes, and the patient's RT was thus hypofractionated, causing him to complete RT in 30 days. This was also 1 of the 3 patients who stopped VPA but not TMZ. All other patients completed RT in 38 to 46 days, accounting for holidays and machine maintenance. Thus, 24 patients finished the prescribed RT/TMZ and VPA according to protocol. Overall 29 patients (78\%) received concurrent RT/TMZ, and 8 patients stopped TMZ as described above. Thirty-three patients (89\%) had adjuvant temozolomide, with a median of 6 cycles.

The most common side effects of RT/TMZ/VPA concomitant treatment were grade 1 and 2 blood/bone marrow toxicity and constitutional, gastrointestinal, and metabolic/laboratory toxicities (Table 2). Grade 3 or 4 toxicity of blood/bone marrow was detected in 12 patients (32\%), and constitutional symptoms in 2 patients (5\%), both of which compared favorably with the previously published RT/TMZ combination (15). With respect to toxicities that 
might have been related to VPA, 3 patients (7\%) had grade 3 metabolic/laboratory toxicity, specifically, an increase in ammonia, and 4 patients (10\%) had grade 3 mental status changes, consistent with the use of high-dose VPA (17).

\section{Time to progression and response}

At the time of this analysis, 28 patients had died. Median duration of follow-up for the entire group was 22 months. Response was evaluated by RECIST criteria (13) at 1 month post-RT with complete history and physical and an MR examination showing $81 \%$ of patients had stable disease and $16 \%$ of patients had progression of disease. For 1 patient (3\%), treatment response was not documented. Response was also retrospectively evaluated by RANO criteria at 1 month post-RT as well as at subsequent follow-up visits for all but 4 patients. Using RANO criteria, we found 2 additional patients who were classified as having had stable disease 1 month post-RT due to radiographic findings which were present within the $80 \%$ RT isodose line, in the absence of clinical progression or histologic confirmation of progression. No change in date of progression was otherwise noted.

On the basis of Kaplan-Meier estimates, the median OS was 29.6 months (95th percentile confidence intervals [CI]: 21-63.8 months) (Fig. 2A). OS rates at 6, 12, and 24 months were 97\% (CI: 92-100), 86\% (CI: 76-98), and 56\% (CI: 42-76), respectively. The median PFS duration was 10.5 months (CI: 7-51 months) as shown in Figure 2B. PFS rates at 6, 12, and 24 months were $70 \%$ (CI: 57-87), 43\% (CI: 30-63), and 38\% (CI: 25-57), respectively. In the cohort that did not progresse to date, the median survival was 33 months (range: 22-62 months).

On univariate analysis, RPA of class 3 versus class $V$ was statistically significant for both improved OS $(P=.019)$ and PFS $(P=.003)($ Fig. 3A/B), younger age ( 50$)$ for both OS $(P=$. $043)$ and PFS ( $P=.051)$ (Fig. 3C and D). VPA sera levels measured in the hospital clinical laboratory were not correlated to survival or to development of grade $3 / 4$ toxicity as shown in Figure 4. The mean VPA concentration in patients without grade 3/4 toxicity was 147 $\mu \mathrm{g} / \mathrm{mL}$, and the mean VPA level in patients with grade $3 / 4$ toxicity was $150 \mu \mathrm{g} / \mathrm{mL}$. Of the 37 patients only 15 of the most recent had MGMT status known with 8 tumors methylated and 7 tumors unmethylated. As expected, patients with methylated tumors had a longer 1year OS and PFS compared to patients with unmethylated tumors (100\% vs $89 \%$ and $78 \%$ vs $30 \%$, respectively).

\section{Discussion}

Despite extensive research into the surgical, radiation and drug treatment options for patients with GBM, prognosis remains poor (1). As RT is the backbone of therapy for GBM there has been much effort in the development of chemoradiation combinations. A number of phase $1 / 2$ trials evaluating chemotherapeutic agents in combination with radiation (reviewed in ref. 7) have been carried out; however, the only phase 3 trial to demonstrate a survival advantage remains the EORTC 26981/22981-NCIC CE3 trial (1), with a median OS of just under 15 months. 
The use of drugs to modify radiation sensitivity in patients with glioblastoma has been extensively studied, with phase $2 / 3$ trials having been performed using halopyrimidines ( 18 , 19), hypoxic cell sensitizers (20-23), anti-angiogenic therapy (24,25), and inhibitors of polyamine synthetase (26), without a significant benefit in either PFS or OS.

However, these phase $2 / 3$ studies were performed before the standard of care became RT plus concurrent TMZ. To compare the toxicity profile from our study to other publications one must deconvolute the causes of the toxicities. For example, in the RT/TMZ phase 2 study by Stupp et al, $15 \%$ of the patients were unable to complete the assigned RT/TMZ with 22 grade 3 and 40 grade 4 toxicities (Supplemental Table; available online at www.redjournal.com) (27). Similarly, in the EORTC 26981/22981-NCIC CE3 phase 3 study, $15 \%$ of the patients did not complete the RT/TMZ, $7 \%$ of the patients had grade $3 / 4$ toxicities, and $32 \%$ of the patients had delays in RT (15). Thus, approximately $15 \%$ of patients did not complete standard TMZ/RT, with a $7 \%$ to $15 \%$ rate of grade $3 / 4$ toxicities. Chakravarti et al (28) studied a radiation modifier without concurrent TMZ and showed that $26 \%$ of patients could not complete the study drug with significant grade 3/4/5 toxicity (28).

For direct comparison to our study, there have been five phase 2 studies that combined a radiation modifier and standard RT/TMZ including three studies of erlotinib, one with enzastaurin and one with poly-ICLC (29-33). In summary, these studies had about $20 \%$ of their patients unable to complete the study drug, fairly significant grade $3 / 4 / 5$ toxicity and unreported delays of RT. Thus, in our study of 37 patients, with $67 \%$ completing study drug, $32 \%$ with a grade $3 / 4 / 5$ and no patient having delays in radiation, the toxicity profile is comparable to the studies using a novel drug/RT/TMZ combination.

The RT/TMZ/VPA protocol resulted in 70\% of patients progression free at 6 months, $86 \%$ of patients alive at 1 year and $56 \%$ of the patients alive at $>2$ years after initiation of therapy. This 6 month progression free survival is comparable to the phase 2 combination studies (Supplementary Table; available online at www.redjournal.com) that had a range of 30-73\% and the phase 3 EORTC study that was $54 \%$. However, the median survival in our trial was 29.6 months and the median survival of the five previous studies ranged from 8.6 to 19.3 months. In addition, since the initiation of our study, retrospective analyses have shown that the use of VPA in conjunction with RT or RT/TMZ may improve the outcome of adult patients with glioblastoma $(34,35)$.

Although results were encouraging, they have several limitations. Because of competition to patient accrual from several large phase 3 trials, our accrual took almost 7 years, during which the treatment of glioblastoma evolved, including the use of RANO criteria for response assessment and the standard assessment of MGMT status. To address these changes, we retrospectively evaluated each patient's response by RANO, and neither PFS nor OS were altered using RANO compared to that using RECIST criteria. Likewise, we evaluated MGMT status on all samples that could be obtained retrospectively. In the samples that we could obtain, there was an even split in our study group between methylated and unmethylated tumors, thus, an over-representation of patients with methylated tumors in our study population is not likely. In addition, the patients in this study had more GTR and a higher RPA class than historical controls. Whether the increased percentage of GTR and 
higher RPA class also contributed to the improvement in outcome observed in this trial cannot be determined but can be tested in a future randomized study. The VPA serum level did not correlate with either outcome or toxicity, similar to the disconnect with the use of VPA as an anti-seizure medication and the suppression of patient seizures. Further development of efficacy biomarkers and advanced tumor pharmacodynamics is thus also needed.

\section{Conclusions}

In summary, with the addition of VPA to standard RT/TMZ therapy, both of the study endpoints were met, with a 1-year OS rate of $86 \%$ (CI: 76-98) and a 6-month PFS rate of $70 \%$ (CI: 57-87). This compares favorably with previously studied combinations and suggests consideration of further investigation in a randomized phase 3 study.

\section{Supplementary Material}

Refer to Web version on PubMed Central for supplementary material.

\section{Acknowledgments}

This work was supported in part by the intramural program of the National Institutes of Health/National Cancer Institute.

\section{References}

1. Stupp R, Hegi ME, Mason WP, et al. European Organisation for Research and Treatment of Cancer Brain Tumour and Radiation Oncology Groups; National Cancer Institute of Canada Clinical Trials Group. Effects of radiotherapy with concomitant and adjuvant temozolomide versus radiotherapy alone on survival in glioblastoma in a randomised phase III study: 5-year analysis of the EORTCNCIC trial. Lancet Oncol. 2009; 10:459-466. [PubMed: 19269895]

2. Senft C, Bink A, Franz K, et al. Intraoperative MRI guidance and extent of resection in glioma surgery: a randomised, controlled trial. Lancet Oncol. 2011; 12:997-1003. [PubMed: 21868284]

3. Gilbert MR, Dignam JJ, Armstrong TS, et al. A randomized trial of bevacizumab for newly diagnosed glioblastoma. N Engl J Med. 2014; 20:699-708. [PubMed: 24552317]

4. Nieder C, Andratschke N, Wiedenmann N, et al. Radiotherapy for high-grade gliomas. Does altered fractionation improve the outcome? Strahlenther Onkol. 2004; 180:401-407. [PubMed: 15241527]

5. Binello E, Green S, Germano IM. Radiosurgery for high-grade glioma. Surg Neurol Int. 2012; 3(suppl 2):S118-s126. [PubMed: 22826815]

6. Waters JD, Rose B, Gonda DD, et al. Immediate post-operative brachytherapy prior to irradiation and temozolomide for newly diagnosed glioblastoma. J Neurooncol. 2013; 113:467-477. [PubMed: 23673513]

7. XXX.

8. XXX.

9. XXX.

10. $\mathrm{XXX}$.

11. XXX.

12. Perucca E. Pharmacological and therapeutic properties of valproate: A summary after 35 years of clinical experience. CNS Drugs. 2002; 16:695-714. [PubMed: 12269862]

13. Therasse P, Arbuck SG, Eisenhauer EA, et al. New guidelines to evaluate the response to treatment in solid tumors. European Organization for Research and Treatment of Cancer, National Cancer 
Institute of the United States, National Cancer Institute of Canada. J Natl Cancer Inst. 2000; 92:205-216. [PubMed: 10655437]

14. Wen PY, Macdonald DR, Reardon DA, et al. Updated response assessment criteria for high-grade gliomas: response assessment in neuro-oncology working group. J Clin Oncol. 2010; 10:19631972. [PubMed: 20231676]

15. Stupp R, Mason WP, van den Bent MJ, et al. European Organisation for Research and Treatment of Cancer Brain Tumor and Radiotherapy Groups; National Cancer Institute of Canada Clinical Trials Group. Radiotherapy plus concomitant and adjuvant temozolomide for glioblastoma. N Engl J Med. 2005; 352:987-996. [PubMed: 15758009]

16. Mirimanoff RO, Gorlia T, Mason W, et al. Radiotherapy and temozolomide for newly diagnosed glioblastoma: recursive partitioning analysis of the EORTC 26981/22981-NCIC CE3 phase III randomized trial. J Clin Oncol. 2006; 24:2563-2569. [PubMed: 16735709]

17. Sztajnkrycer MD. Valproic acid toxicity: overview and management. J Toxicol Clin Toxicol. 2002; 40:789-801. [PubMed: 12475192]

18. Phillips TL, Levin VA, Ahn DK, et al. Evaluation of bromodeoxyuridine in glioblastoma multiforme: a Northern California Cancer Center phase II study. Int J Radiat Oncol Biol Phys. 1991; 21:709-714. [PubMed: 1651306]

19. Urtasun RC, Cosmatos D, Del Rowe J, et al. Iododeoxyuridine (IUdR) combined with radiation in the treatment of malignant glioma: a comparison of short versus long intravenous dose schedules (RTOG 86-12). Int J Radiat Oncol Biol Phys. 1993; 27:207-214. [PubMed: 8407393]

20. Kleinberg LL, Grossman SA, Carson K, et al. Survival of patients with newly diagnosed glioblastoma multiforme treated with RSR13 and radiotherapy: Results of a phase II new approaches to brain tumor therapy CNS consortium safety and efficacy study. J Clin Oncol. 2002; 20:3149-3155. [PubMed: 12118029]

21. Nelson DF, Schoenfeld D, Weinstein AS, et al. A randomized comparison of misonidazole sensitized radiotherapy plus BCNU and radiotherapy plus BCNU for treatment of malignant glioma after surgery; preliminary results of an RTOG study. Int J Radiat Oncol Biol Phys. 1983; 9:1143-1151. [PubMed: 6347995]

22. Del Rowe J, Scott C, Werner-Wasik M, et al. Single-arm, open-label phase II study of intravenously administered tirapazamine and RT for glioblastoma multiforme. J Clin Oncol. 2000; 18:1254-1259. [PubMed: 10715295]

23. Miralbell RO, Mornex F, Greiner R, et al. Accelerated radiotherapy, carbogen, and nicotinamide in glioblastoma multiforme: report of European Organization for Research and Treatment of Cancer trial 22933. J Clin Oncol. 1999; 17:3143-3149. [PubMed: 10506611]

24. Lai A, Tran A, Nghiemphu PL, et al. Phase II study of bevacizumab plus temozolomide during and after radiation therapy for patients with newly diagnosed glioblastoma multiforme. J Clin Oncol. 2011; 29:142-148. [PubMed: 21135282]

25. Hainsworth JD, Shih KC, Shepard GC, et al. Phase II study of concurrent radiation therapy, temozolomide, and bevacizumab followed by bevacizumab/everolimus as first-line treatment for patients with glioblastoma. Clin Adv Hematol Oncol. 2012; 10:240-246. [PubMed: 22706484]

26. Prados MD, Wara WM, Sneed PK, et al. Phase III trial of accelerated hyperfractionation with or without difluromethylornithine (DFMO) versus standard fractionated radiotherapy with or without DFMO for newly diagnosed patients with glioblastoma multiforme. Int J Radiat Oncol Biol Phys. 2001; 49:71-77. [PubMed: 11163499]

27. Stupp R, Dietrich PY, Ostermann Kraljevic S, et al. Promising survival for patients with newly diagnosed glioblastoma multiforme treated with concomitant radiation plus temozolomide followed by adjuvant temozolomide. J Clin Oncol. 2002; 20:1375-1382. [PubMed: 11870182]

28. Chakravarti A, Wang M, Robins HI, et al. RTOG 0211: A phase $1 / 2$ study of radiation therapy with concurrent gefitinib for newly diagnosed glioblastoma patients. Int J Radiat Oncol Biol Phys. 2013; 85:1206-1211. [PubMed: 23182702]

29. Brown PD, Krishnan S, Sarkaria JN, et al. North Central Cancer Treatment Group study N0177. Phase I/II trial of erlotinib and temozolomide with radiation therapy in the treatment of newly diagnosed glioblastoma multiforme. J Clin Oncol. 2008; 26:5603-5609. [PubMed: 18955445] 
30. Peereboom DM, Shepard DR, Ahluwalia MS, et al. Phase II trial of erlotinib with temozolomide and radiation in patients with newly diagnosed glioblastoma multiforme. J Neurooncol. 2010; 98:93-99. [PubMed: 19960228]

31. Prados MD, Chang SM, Butowski N, et al. Phase II study of erlotinib plus temozolomide during and after radiation therapy in patients with newly diagnosed glioblastoma multiforme or gliosarcoma. J Clin Oncol. 2009; 27:579-584. [PubMed: 19075262]

32. Rosenfeld MR, Chamberlain MC, Grossman SA, et al. A multi-institution phase II study of polyICLC and radiotherapy with concurrent and adjuvant temozolomide in adults with newly diagnosed glioblastoma. Neuro Oncol. 2010; 12:1071-1077. [PubMed: 20615924]

33. Butowski N, Chang SM, Lamborn KR, et al. Phase II and pharmacogenomics study of enzastaurin plus temozolomide during and following radiation therapy in patients with newly diagnosed glioblastoma multiforme and gliosarcoma. Neuro Oncol. 2011; 13:1331-1338. [PubMed: 21896554]

34. Weller M, Gorlia T, Cairncross JG, et al. Prolonged survival with VPA use in the EORTC/NCIC temozolomide trial for glioblastoma. Neurology. 2011; 77:1156-1164. [PubMed: 21880994]

35. Barker CA, Bishop AJ, Chang M, et al. Valproic acid use during radiation therapy for glioblastoma associated with improved survival. Int J Radiat Oncol Biol Phys. 2013; 86:504-509. [PubMed: 23523186] 


\section{Summary}

This is a report of a phase 2 trial of 37 patients with newly diagnosed glioblastoma treated with valproic acid (VPA) at a dose of $25 \mathrm{mg} / \mathrm{kg}$ divided into 2 daily doses, concurrent with radiation and temozolomide therapy. VPA toxicity was comparable with historical data. Median overall survival and progression-free survival were 29.6 months and 10.5 months, respectively. 


\section{$10-15 \rightarrow 25 \mathrm{mg} / \mathrm{kg} / \mathrm{day}$}

VPA

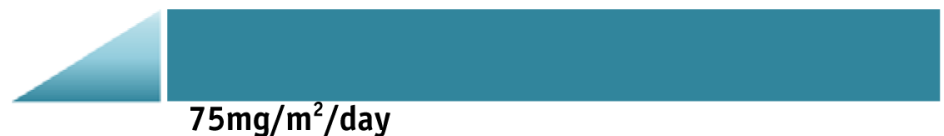

TMZ

RT

Weeks

\section{$75 \mathrm{mg} / \mathrm{m}^{2} /$ day}

$$
2 \mathrm{~Gy} / \mathrm{day}
$$

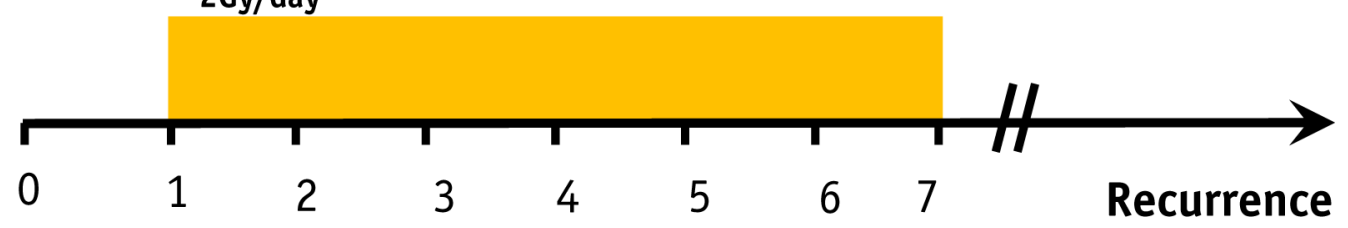

Fig. 1.

Treatment schema for the study group. VPA was administered concurrently with RT. No adjuvant VPA was given. $\mathrm{RT}=$ radiation therapy; VPA = valproic acid. 

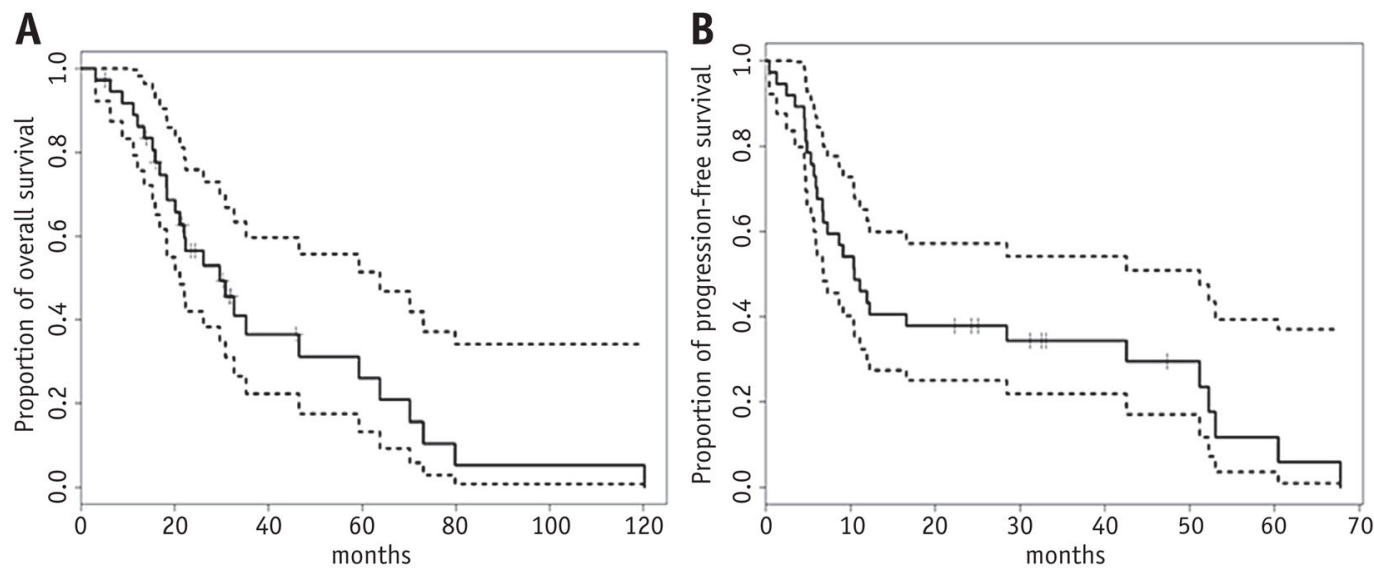

Fig. 2.

Kaplan-Meier analysis of overall survival (A) and progression-free survival (B). Dotted lines represent $95 \%$ confidence limits. 

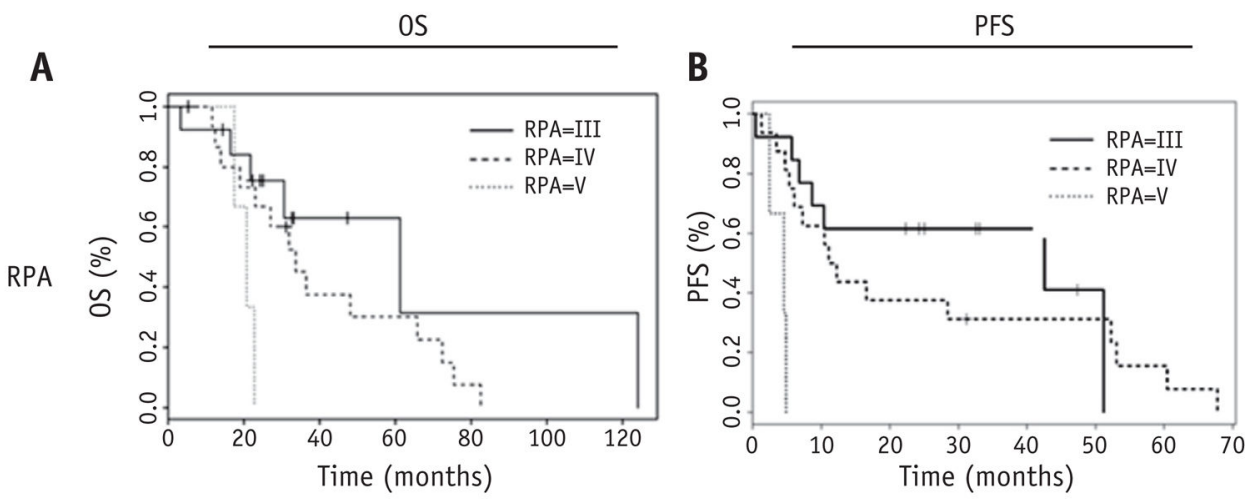

C

D
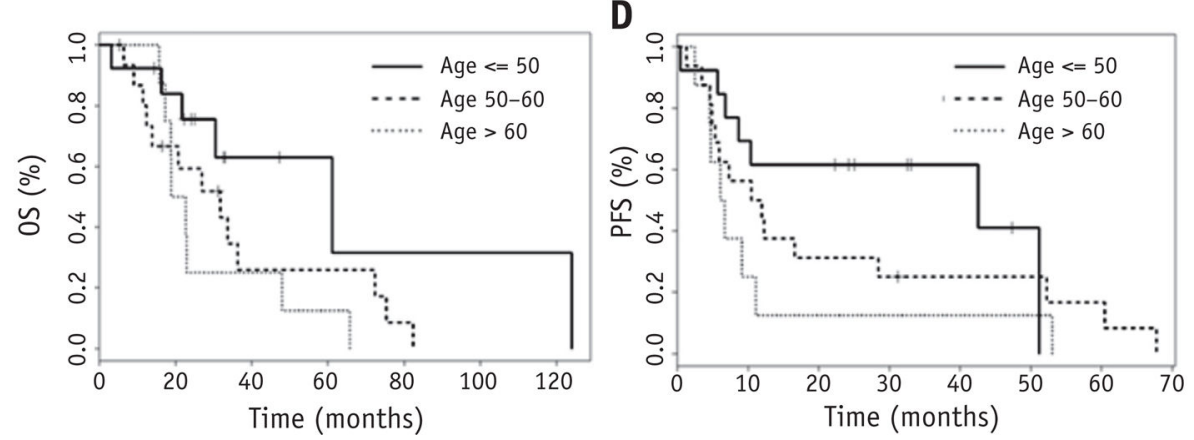

Fig. 3.

Prognostic factor analysis: Kaplan-Meier analysis of overall survival (OS) and progression free survival (PFS) by RPA (A and B) and age (C and D). RPA $=\mathrm{XXX}$. 
Survival vs highest VPA level

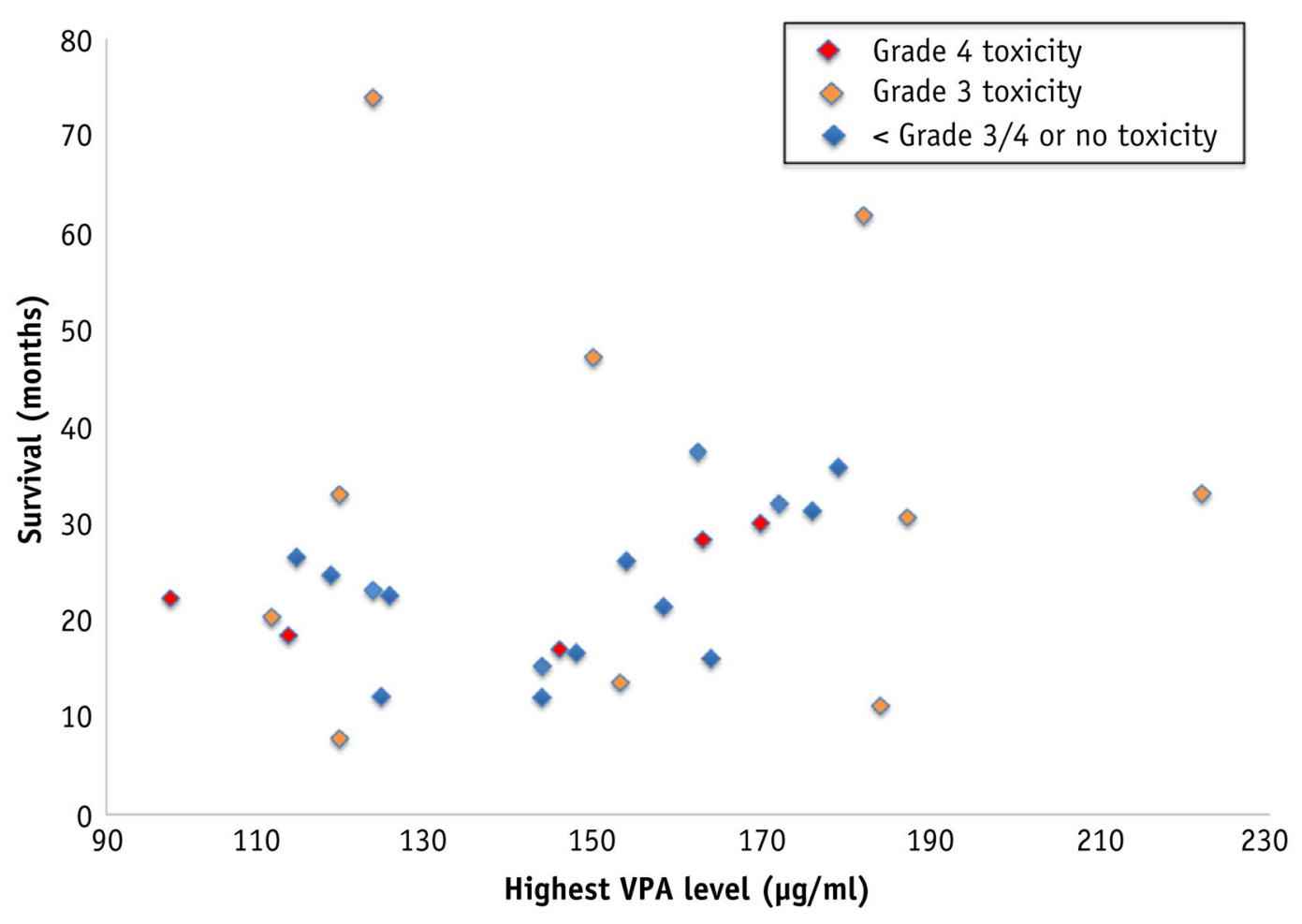

Fig. 4.

Survival and toxicity versus VPA levels (according to possible or probable attribution to VPA) for patients in whom VPA levels were available. VPA = valproic acid. 


\section{Table 1}

Valproic acid study pretreatment characteristics

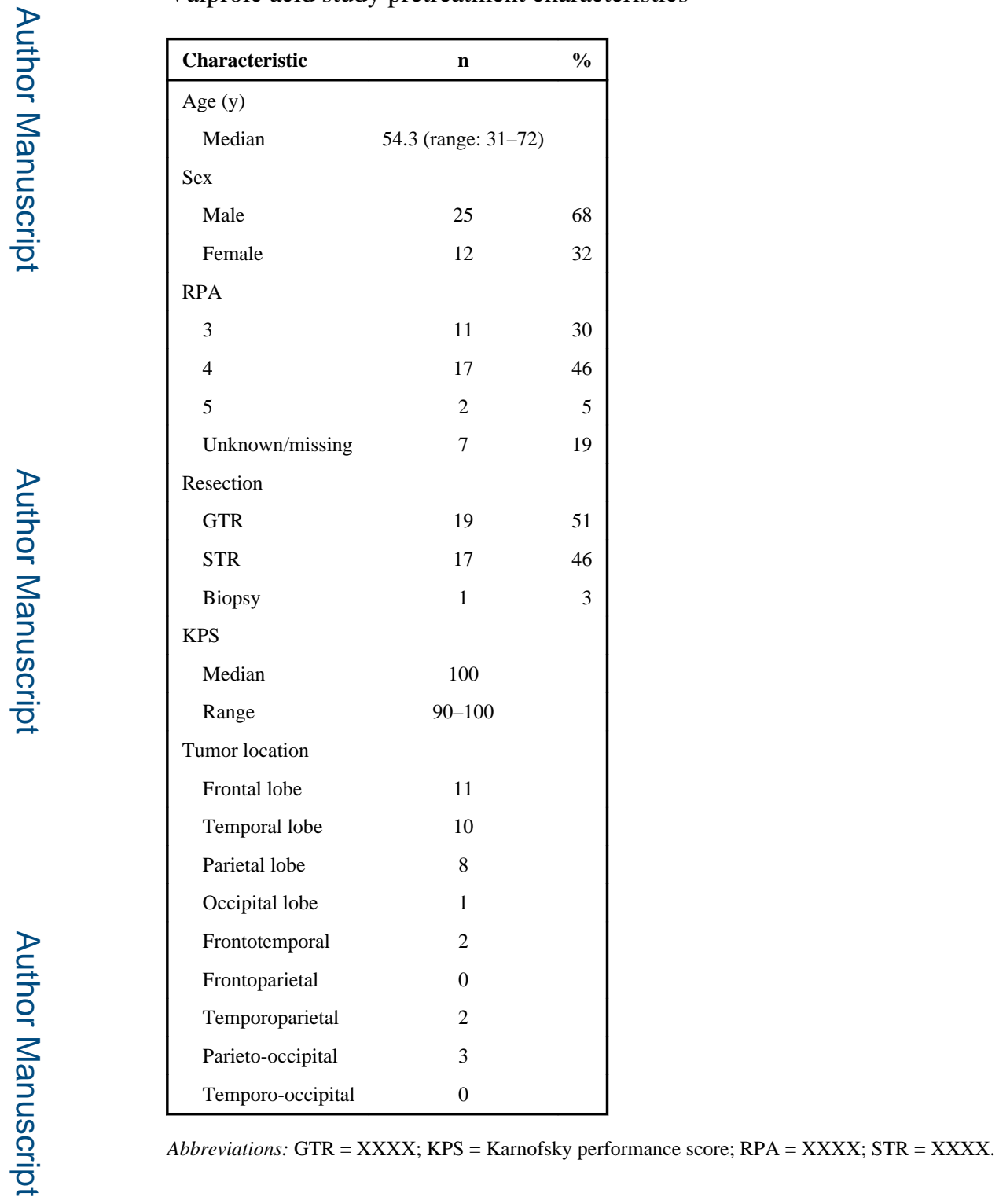




\section{Table 2}

Acute toxicity related to temozolomide, valproic acid, and radiation therapy

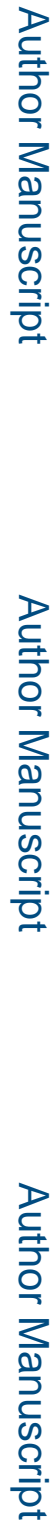

\begin{tabular}{|lrrrr|}
\hline & \multicolumn{5}{c|}{ Grade } \\
\cline { 2 - 5 } Toxicity & $\mathbf{1}$ & $\mathbf{2}$ & $\mathbf{3}$ & $\mathbf{4}$ \\
\hline Auditory/hearing & 1 & 7 & 0 & 0 \\
Blood/bone marrow & 8 & 9 & 6 & 6 \\
Cardiovascular & 2 & 0 & 0 & 0 \\
Coagulation & 1 & 0 & 0 & 0 \\
Constitutional symptoms & 20 & 3 & 2 & 0 \\
Dermatology/skin & 21 & 3 & 0 & 0 \\
Endocrine & 0 & 1 & 0 & 0 \\
Gastrointestinal & 21 & 4 & 0 & 0 \\
Hepatobiliary/pancreas & 2 & 0 & 0 & 0 \\
Lymphatics & 2 & 2 & 0 & 0 \\
Metabolic/laboratory & 16 & 14 & 3 & 0 \\
Musculoskeletal & 1 & 1 & 0 & 0 \\
Neurology & 5 & 11 & 4 & 0 \\
Ocular/visual & 6 & 0 & 1 & 0 \\
Pain & 7 & 2 & 0 & 0 \\
\hline
\end{tabular}

로를

를

Int J Radiat Oncol Biol Phys. Author manuscript; available in PMC 2016 August 01. 